by the publishers at the end of 1966 , does not suffer from these faults.

\title{
S-MATRIX PRIMER
}

Contributions to the Theory of Weak and Strong Interactions of Elementary Particles

Edited by V. B. Berestetskii. Translated from the Russian. (Lectures read at the Spring School of Theoretical and Experimental Physics, Nor-Amberd, 3-14 April, 1961.) Pp. vi +170 . (Jerusalem: Israel Program for Scientific Translations; London: Oldbourne Press, 1965.) $72 s$.

IT would seem profitless to translate a book in a rapidly changing field of physics five years after its publication and especially when it is a collection of lecture notes by different authors. The excuse for such notes is that they make up in topicality what they lack in cohesion and careful exposition. These lectures, however, given at Nor-Amberd in 1961, are good enough to preclude any such simple conclusion. While the few which are mainly concerned with symmetry properties of elementary particles have been overtaken by ideas such as $S U 3$ symmetry and the Cabibbo theory, the core of the book forms a primer on $S$-matrix theory which still has value.

The lectures give a concise connected discussion of the use of dispersion relations and unitarity in elementary particle dynamics, from the Kramers-Krönig theory of photon scattering to applications of the Mandelstam representation. The emphasis is on the mathematical techniques rather than the deep physical ideas and approximations; the exposition is generally clear throughout, but I found the lectures by Gribov and ter Martirosyan particularly good. The drawbacks of antiquity appear here too: Regge poles are not mentioned, but the omission is not as serious as it may sound in that there are several books available complementary to these lectures; the reader will have to find them himself, however, for the references have not been brought up to date.

With these reservations, the book can be recommended to theoretical research students as an introduction to "first generation" $S$-matrix theory. The translation by Zvi Lerman is crisp, though not always idiomatic. The price is in line with current trends but includes a hard cover.

$$
\text { Hugh Burkhardt }
$$

\section{MECHANICS, CLASSICAL AND QUANTUM}

Classical Mechanics, Quantum Mechanics, Field Theory (Pure and Applied Physics: a Series of Monographs and Textbooks.) By Amnon Katz. Pp. $x+207$. (New York: Academic Press, Inc. ; London: Academic Press, Inc. (London), Ltd., 1965.) 60s.

THIs book is the twenty-second in a series of monographs and text-books. Its four chapters lead from classical mechanics through quantum mechanics, including many particle systoms, to the elements of relativistic theory. The emphasis is on a unified approach to classical and quantum physies, the passage from one to the othor being through action principles and functional integration. Inevitably there is a certain loss of rigour in manipulation of the integrals, but apart from this the exposition is clear and well set out and provides a good overall picture of the subject matter.

This is not a book to be used on its own by the beginner in quantum mechanics. The approach is useful as a unification but is both conceptually and notationally too complex for the average novice. There are few standard examples worked and none left as exercises. The hydrogen

atom and harmonic oscillator are ignored. In conjunction with the standard text, however, or used by those with a working knowledge of the basic material of classical and quantum mechanics, this book could provide a valuable insight into the relationship between the two systems.

David Harding

\section{NUCLEAR STRUCTURE}

\section{Nuclear Structure Study with Neutrons}

Edited by M. Nève de Mévergnies, P. Van Assche and $J$. Vervier. (Proceedings of the International Conference on the Study of Nuclear Structure with Neutrons, Antwerp, 19-23 July 1965.) Pp. vi +588. (Amsterdam: North-Holland Publishing Company, 1966.) $120 s$.

AT a recent conference, it was estimated that both the number of active physicists and their publications are increasing exponentially with a time-constant of 17 years. Presumably conferences themselves will follow a similar law. The number of conferences on nuclear physics in the past decade has increased significantly with a marked tendency towards specialization.

This volume, which covers the proceedings of one such specialist conference, includes papers by invited speakers and by rapporteurs, together with abstracts of the many contributed papers. Seven sessions aro reported under the headings-nuclear models and the neutron-nucleon interaction; nuclear spectroscopy; neutron resonances and strength functions; intermediate structure and the statistical model; neutron capture mechanisms and photoneutron reactions; optical model; special topies. The invited papers and the rapporteurs' accounts are authoritative and of current interest. Throughout, one is impressed by the quality of the experimental data being produced by time-of-flight neutron spectroscopy, large volume germanium-lithium $\gamma$-detectors, polarized neutron sources, etc., and on the theoretical side by the continuing success of nuclear models, ever more sophisticated. The whole adds up to a wide coverage of neutron physics and this book must be of interest to all nuclear physicists, not just those concerned with neutrons.

The abstracts of the contributed papers are only partially grouped according to subject, which is unfortunate because there is no index. One criticism of editorial policy is that to save space only abstracts of the few short contributions actually read at the conference are given in the main text. As a result the value of the discussion, given verbatim, is reduced. For those interested, full details of all the short contributions are available in a separate publication, EANDC-45-S, in two volumes.

\section{J. M. Calvert}

\section{INTERNAL CONVERSION}

\section{Internal Conversion Processes}

(Including the Proceedings of the International Conference on the Internal Conversion Process, May 10-13, 1965, Vanderbilt University, Nashville, Tennessee.) Edited by Joseph H. Hamilton. Pp. xxv +669 . (New York: Academic Press, Inc.; London: Academic Press, Inc. (London), Ltd., 1966.) $180 s$.

THIs book consists largely of the proceedings of a conference on internal conversion processes held in Nashville, Tennessee, in 1965, and as such conference proceedings go it is well produced. Contributors havo been allowed enough space to make an adequate report of their work, and the invited review papers preceding the various sessions are extremely useful. However, no doubt realizing the rather limited value such proceedings usually have, the conference organizers or perhaps the editor have included 